\title{
A Proposed Orthogonal Chirp Division Multiplexing (OCDM) Multicarrier Transceiver Based on the Discrete Fractional Cosine Transform
}

\author{
Hani Attar'1, Ahmed Solyman² \\ ${ }^{1}$ Department of Electronic and Communication Engineering, Philadelphia University, Amman, Jordan \\ ${ }^{2}$ The Military Technical Research Center, Cairo, Egypt \\ Email: attar_hani@hotmail.com, Aaasolyman@gmail.com
}

How to cite this paper: Attar, H. and Solyman, A. (2017) A Proposed Orthogonal Chirp Division Multiplexing (OCDM) Multicarrier Transceiver Based on the Discrete Fractional Cosine Transform. Journal of Computer and Communications, 5, 34-47. https://doi.org/10.4236/jcc.2017.52005

Received: December 19, 2016

Accepted: February 21, 2017

Published: February 24, 2017

Copyright $\odot 2017$ by authors and Scientific Research Publishing Inc. This work is licensed under the Creative Commons Attribution International License (CC BY 4.0).

http://creativecommons.org/licenses/by/4.0/

\begin{abstract}
Doppler spread is a phenomenon caused by rapid changes in the channel response due to the movement of the transmitter/receiver through a multipath environment, which is also known as a doubly selective channel response. In doubly selective fading channel where the channel frequency response is regarded as rapidly time-varying, the inter carrier interference (ICI) occurs mainly because the traditional multicarrier transceivers are incapable to diagonalize the channel matrix. The proposed scenario is for an Orthogonal Chirp Division Multiplexing (OCDM) multicarrier system derived from Discrete Fractional Cosine Transform (DFrCT). The proposed DFrCT-OCDM multicarrier system is proofed to be less signal processing complexity and easier to implement, compared to the traditional Fast Fourier Transform (FFT). Simulation results reveal that the DFrCT provides superior performance reflected the significant improvement in the BitError Rate which reaches to $10 \mathrm{~dB}$, better spectrum efficiency and better energy concentration properties than the conventional FFT. Finally, several equlizers are applied and they all show that the proposed DFrCT-OCDM over performs the other systems with all these equlizers.
\end{abstract}

\section{Keywords}

DFT, DFrCT, OFDM, Doubly Selective Channels, Doppler Spread

\section{Introduction}

The origin of Orthogonal Frequency Division Multiplexing (OFDM) development was introduced as early as 1950's with the introduction of Frequency Divi- 
sion Multiplexing (FDM) for data communications in early telephone lines. FDM divides the channel bandwidth into sub-channels and transmits multiple relatively low rate signals by carrying each signal on a separate carrier frequency. Guard-bands are left between the different sub-channels to ensure elimination of the Inter-Symbol-Interference (ISI) and separation between sub-channels, which led to bandwidth inefficiency [1] [2] [3] [4].

Doppler spread is a phenomenon caused by rapid changes in the channel response due to movement of the transmitter/receiver through a multipath environment which is also known as a doubly selective channel response. The amount of Doppler spread is proportional to the transmission frequency and the velocity of movement. The closer the sub-carriers are spaced together, the more OFDM signal is effected by the Doppler spread, thus a trade-off exists between the amount of multipath protection (length of the guard period) which is bandwidth inefficient and the Doppler spread tolerance, which is needed to reduce the Doppler spread effect, i.e. when increasing the mobile communication speed; the Doppler spread frequency increases resulting to the need for a longer guard period between the sub-carriers, which is regarded as bandwidth waste [4]-[10].

Moreover, the channels capacity and performance can be limited due to the multipath propagation problems, resulting to frequency selective fading response [8].

To fulfill the desires for high speed wireless communication at such high data rates transmission over delay-dispersive channels; the OFDM is widely implemented over delay-dispersive channels, which is a Multi-Carrier Modulation (MCM) scheme [10] [11] [12].

If only a single frequency band (single carrier) is used, then the symbol duration $\left(T_{S}\right)$ has to be very small in order to achieve the required data rate, when symbol duration $T_{S}$ is not larger than the channel delay spread $(\tau)$, ISI occurs and the communication systems will not be able to achieve good system performance [7]. Moreover, a very small symbol duration has a very long bandwidth, thus requires a complicated equaliser (mathematically expensive) with a large number of taps at the receiver and increases the computational effort [1] [2].

OFDM converts the high data rate stream into a number of low-data-rate streams (sub-channels) $N$ and modulates each one of the low-data-rate streams over one of the sub-channels. These are then transmitted at the same time keeping the sum of the desired data rate constant, with the property that each of the sub-channels has $T_{S} \gg \tau$ and hence effectively ISI-free channels are obtained and the multipath fading channel can be seen as flat fading channel for each subcarrier (sub-channels experience relatively flat fading) as shown in Figure 1.

The sub-channels are orthogonal under ideal propagation conditions. Moreover ISI can be completely eliminated through the use of a cyclic prefix [13].

Traditional OFDM multicarrier technique is used to diagonalize the frequency domain for the frequency-selective channel matrix. Moreover, the single tap equalizer is implemented to compensate the distortion that affects each independent sub-channel through simple gain and phase adjustment [1] [2].

Digital Fourier Transform (DFT) is performed to enable implementing OFDM 
and even makes it over performs the well-implemented single carrier systems. However, in doubly selective rapidly fading channels, such as the channels for the modern wireless systems, like Digital Video Broadcasting Handheld (DVB$\mathrm{H}$ ) and the high speed (beyond $100 \mathrm{~km} / \mathrm{h}$ ) mobile broadband services providers (microwave access WiMAX); it is regarded as less effective [3] [4] [5] [6] [7].

The applications mentioned above require to use OFDM in doubly dispersive channel (time-frequency fading channels) environments resulting to the lost in the orthogonallity of the sub-carrier, which causes the inter-carrier interference (ICI) [7] [8] [9] [10] [11].

To mitigate the change in the channel behaviour at high speed, i.e. the rapidly time-varying frequency response; we propose to make the sub-channel carrier frequencies to be time-varying as well, in order to cope with the distortion caused by the carriers channels. Applying Discrete Fractional Cosine Transform (DFrFT) instead of Fast Fourier Transformer (FFT) in multicarrier systems is well investigated in [11] [12] [14] and the references their-in, mainly to minimize the negative impact of Doppler frequency spreads. unlike the work performed in this paper, where Orthogonal Chirp Division Muliplex (OCDM) is implemented and fully investigated, hence the consequential DFrFT-OCDM system consists of orthogonal chirp signals of carriers, in the form of a block transfer system.

The chirp signal ideally is regarded as a frequency modulated signal which sweeps over a wide spectrum without any changing in its amplitude as shown in Figure 2.

The chirp signal uses a wideband spectrum for transmission and its main advantage that its resistant to the negative channel effects, such as the noise, multipath fading and Doppler effects [15].

In this paper we present a novel OCDM system that employs the Discrete Fractional Cosine Transform (DFrCT) basis as multicarrier system subcarriers. Unlike [12]-[17], where DFrCT based on the eigen-decomposition of DCT kernel was proposed. Moreover, the proposed scenario in this paper for the transformation is discrete and strictly invertible with less computation complexity, better spectral efficiency and energy concentration than the DFrFT, with maintaining the principle of using the same chirp like (time-frequency) signal properties. Furthermore, as will be shown in this paper, the complexity of the DFrCT-



Figure 1. Received OFDM signal in the frequency domain. 


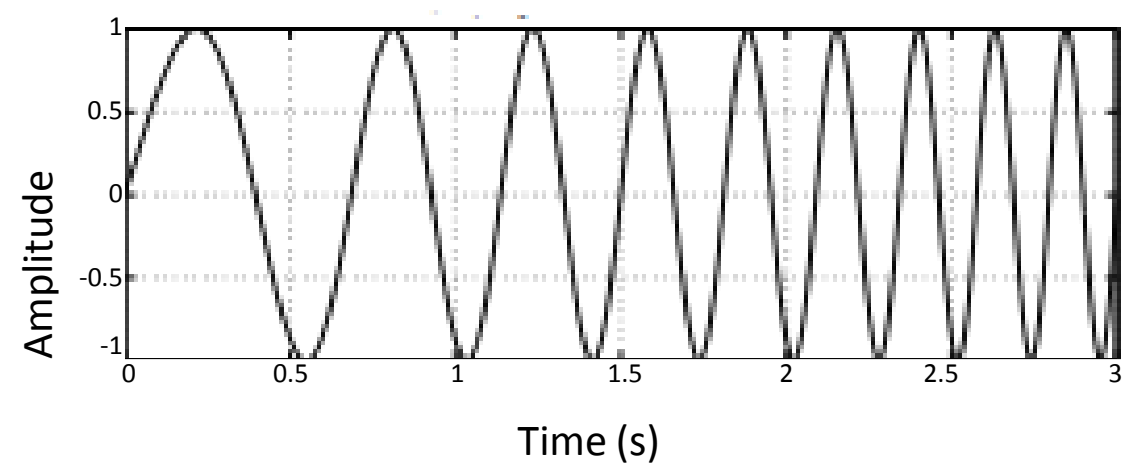

Figure 2. Illustration of chirps: A chirp signal from 1 to $3 \mathrm{~Hz}$.

OCDM system is actually lower than that of conventional OFDM systems employing the DFT.

In [18], Peak-to-Average power ratio performance with BER analysis for Orthogonal Chirp Division Multiplexing Multicarrier Systems Based on Discrete Fractional Cosine Transform, and it showed how it is possible to improve the performance when using DFCT.

The rest of the paper is organized as follows: In Section 2, the Discrete Fractional Cosine Transform is introduced. In Section 3, the system model is presented and a description of the key realization of the transceiver is provided. In Section 4 the results of comparing the proposed system (DFrCT-OCDM) and the conventional OFDM and FrFT-OCDM systems are shown. Finally, Section 5 concludes the paper.

\section{The Fractional Cosine and Sine Transform}

The FrFTis defined in [16] as an angle $\alpha$ rotation on the time frequency distribution, accordingly, for $\alpha=0$, there will be no change after applying DFrFT, and for $\alpha=\pi / 2$, DFrFT becomes a Fourier transform, which rotates the time frequency distribution with $\pi / 2$. For other values of $\alpha$, the DFrFT rotates the time frequency distribution according to $\alpha$ [19].

Figure 3 illustrates the relationships between the time, and frequency outputs from the DFrFT $\left(F_{\alpha}\right)$ when the fractional angle $\alpha$ changes as:

$$
\boldsymbol{F}_{\alpha}=\left\{\begin{array}{lll}
\boldsymbol{F}_{0}[x(t)]=x(t) & \text { for } & \alpha=0 \underline{\text { yields Timedomain }} \\
\boldsymbol{F}_{\pi / 2}[x(t)]=X(f) & \text { for } & \alpha=\pi / 2 \underline{\text { yields }} \text { Frequencydomain } \\
\boldsymbol{F}_{\pi}[x(t)]=x(-t) & \text { for } & \alpha=\pi \underline{\text { yields Inversetimedomain }} \\
\boldsymbol{F}_{3 \pi / 2}[x(t)]=X(-f) & \text { for } & \alpha=3 \pi / 2 \underline{\text { yields Inversefrequencydomain }}
\end{array}\right.
$$

It can also be seen from Figure 3 as well that $\boldsymbol{F}_{2 \pi}[x(t)]=\boldsymbol{F}_{0}[x(t)]=x(t)$ [19].

Based on Figure 3; the FrFT is considered as a fractional power of the Fourier transform operator, where in the frequency-time plane; $f(t)$ is the original signal in the time domain and $F_{\alpha}(u)$ is equivalent to $\alpha^{\text {th }}$ order in the fractional domain. 


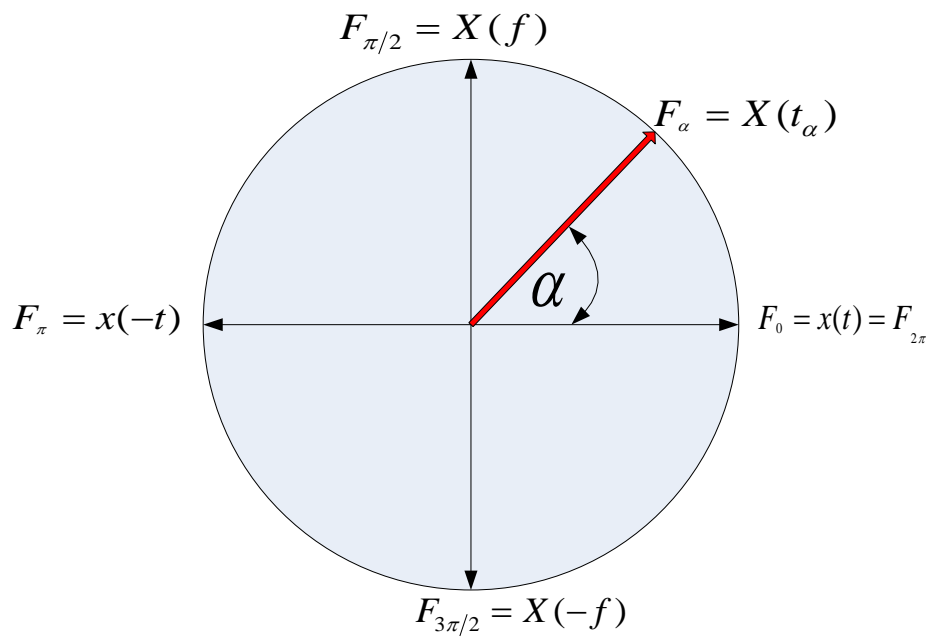

Figure 3. Signal $x(t)$ at different transformation angle $\alpha$ in the frequency-time plane.

Discrete Cosine Transformer (DCT) and Discrete Sine Transformer (DST) can be considered as a generalization of the cosine and sine transform respectively, where the $\alpha^{\text {th }}$ order fractional cosine transform (FrCT) is $F_{\alpha}^{c}(u)$ and the $\alpha^{\text {th }}$ order fractional sine transform (FrST) is $F_{\alpha}^{c}(u)$ shown in Equations (1) and (2) from [19] [20] [21]:

$$
\begin{aligned}
& F_{\alpha}^{c}(u)=A_{\alpha} e^{j\left(u^{2} / 2\right) \cot \cot \alpha} \int_{-\infty}^{\infty} \cos (\csc \csc \alpha \cdot u t) e^{j\left(t^{2} / 2\right) \cot \cot \alpha} f(t) \mathrm{d} t \\
& F_{\alpha}^{s}(u)=A_{\alpha} e^{j(\alpha-\pi / 2)} e^{j\left(u^{2} / 2\right) \cot \alpha} \int_{-\infty}^{\infty} \sin (\csc \alpha \cdot u t) e^{j\left(t^{2} / 2\right) \cot \alpha} f(t) \mathrm{d} t
\end{aligned}
$$

where $\alpha=-\frac{\pi}{2}$ to $\frac{\pi}{2}$, is the angle between the fractional order axis $u$ and time axis $t$, and $A_{\alpha}=\sqrt{\frac{1-j \cot \alpha}{2 \pi}}$.

Various definitions of the DFrCT differ in accuracy and complexity and may be derived using extensions from the FrFT by sampling the kernel real/ imaginary parts of FrFT or directly from the DCT itself [14]. The DFrCT definition in [19] is used in our work as it has minimal complexity and has a simple inverse transform. In the following we will denote the DFrCT as $F_{\alpha}=F_{\alpha}^{c}$ in [19] and the DFrCT uses the DFT Hermite eigenvector decomposition and the DCT transform kernel.

The vector notation for the DFrCT is given by Equation (3):

$$
X=\left[\begin{array}{c}
X_{\alpha}(0) \\
X_{\alpha}(1) \\
\vdots \\
X_{\alpha}(N-1)
\end{array}\right]=F_{\alpha}\left[\begin{array}{c}
x(0) \\
x(1) \\
\vdots \\
x(N-1)
\end{array}\right]=F_{\alpha} \cdot x
$$

where $F_{\alpha}$ is the unitary $N \times N$ DFrCTmatrix, $N$ is the number of samples and $\alpha$ is the transform rotational angle represented in the time-frequency plane shown in Figure 4. Note that when $\alpha=\pi / 2$, the DFrCT will become the 


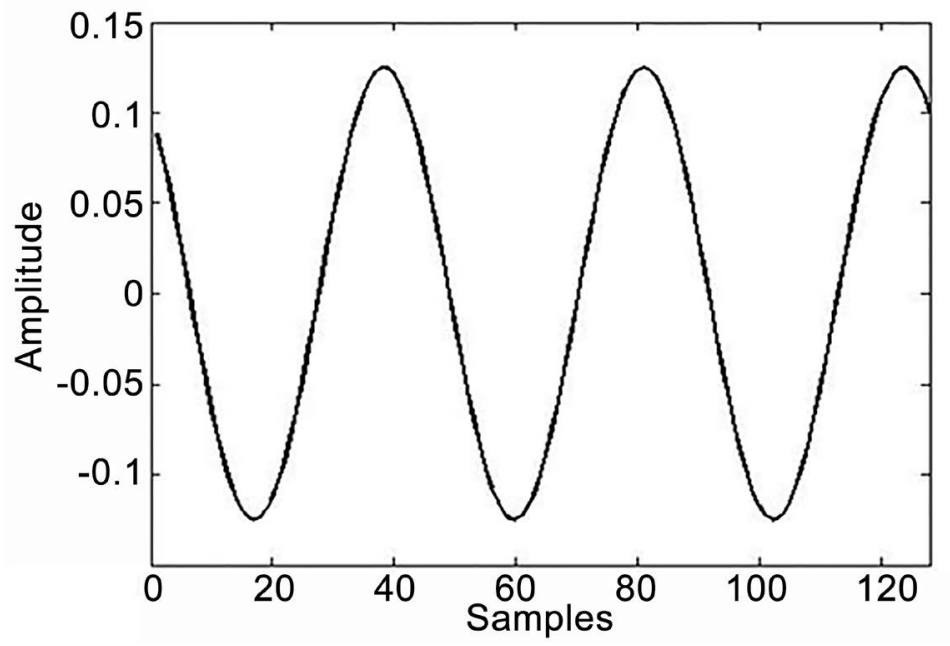

(a) DFT-OFDM Subcarriers

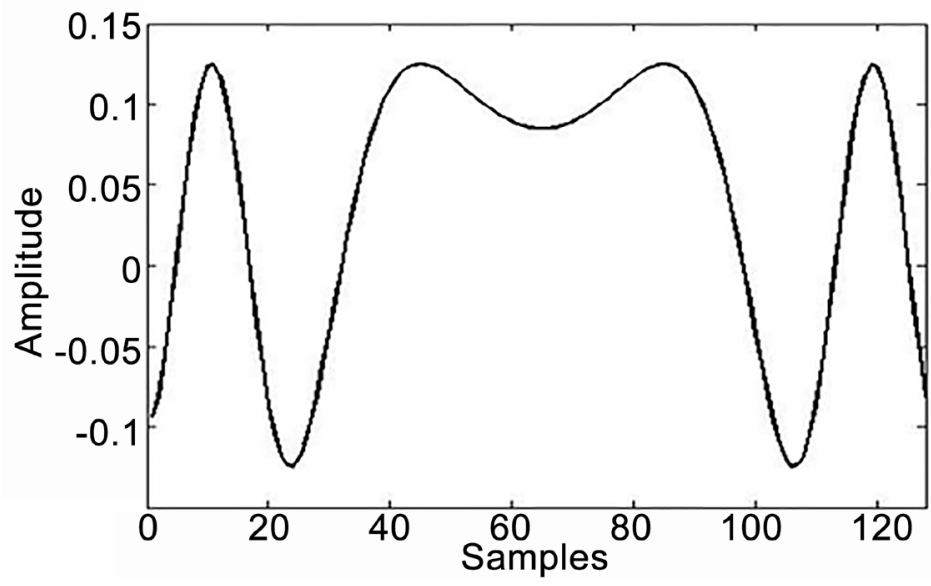

(b) DFrFT-OCDM Subcarriers

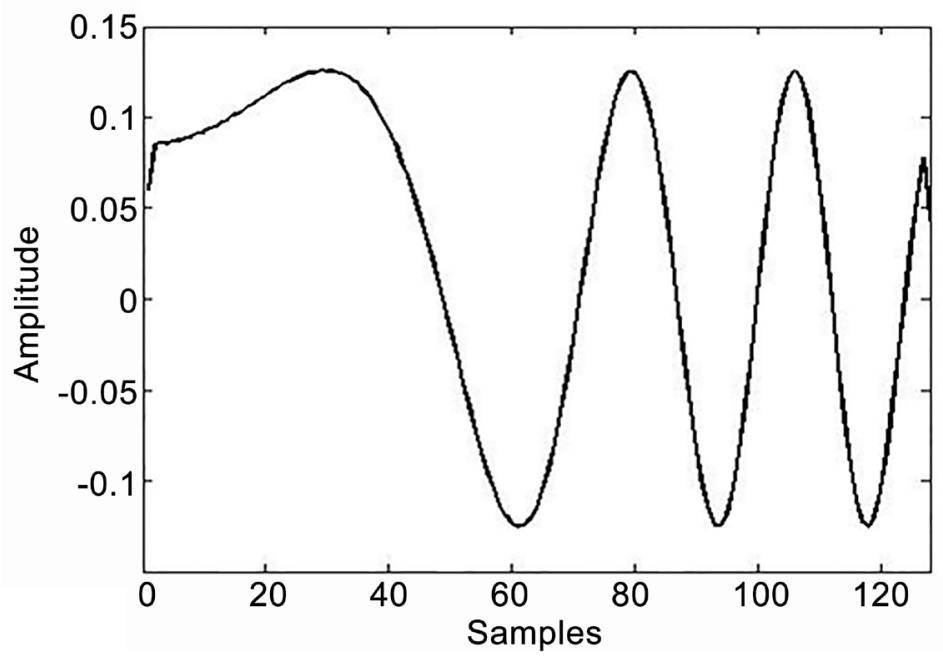

(c) DFrCT-OCDM Subcarriers

Figure 4. DFrCT, DFrFT-OCDM with $\alpha=0.95 \frac{\pi}{2}$ and DFT-OFDM Subcarrier 2 (a) DFT-OFDM; (b) DFrFT-OCDM and (c) DFrCT-OCDM. 
conventional DCT and when $\alpha=0, F_{\alpha}$ is an identity matrix [19]. Similarly the inverse DFrCT (IDFrCT) is given in Equation (4):

$$
x=F_{-\alpha} \cdot X
$$

where $F_{-\alpha}=F_{\alpha}^{H}$ and $(.)^{H}$ denotes the complex conjugate transpose operation.

\section{DFrCT Complexity}

Implementing the FrFT for a given signal requires one DFT and two chirp multiplications, since an efficient DFT requires approximately $(P / 3) \cdot \log _{2} P$ complex multiplications (using the split-radix algorithm) where $\mathrm{P}$ is the total number of sampling points. Therefore a total of approximately $2 P+(P / 3) \cdot \log _{2} P$ Complex Multiplications (CMult) are required to implement the FrFT [14].

The complex number multiplication CMult requires a minimum of three folders more than the com required in the real number multiplications, resulting to Equation (5) which gives the amount of real number multiplications required for the FrFT:

$$
\operatorname{FrFT}_{\mathrm{C}_{\text {Mult }}}=6 P+P \cdot \log _{2} P
$$

where $F r F T_{C_{\text {Mult }}}$ is the FrFT complex multiplication number.

Using similar arguments, when computing the DFrCT from the first-type DCT kernel [14], the required complex multiplication number for DFrCTis given in Equation (6):

$$
\operatorname{DFrCT}_{C_{\text {Mult }}}=2 P+(P / 2) \cdot \log _{2}(P / 2)
$$

where $\operatorname{DFrCT}_{C_{\text {Mut }}}$ is the DFrCT complex multiplication number.

Based on above and by comparing Equation (5) with Equation (6); it is obvious that when implementing FrCT; the system complexity is approximately decreased to the half when compared with the complexity of the FrFT.

\section{The DFrCT Based OCDM System Model}

\subsection{The DFrCT-OCDM Bases}

A comparison between the DFrCT, DFrFT-OCDM and OFDM real part of Subcarrier 2 are shown in Figure 4 where $\alpha=0.95 \frac{\pi}{2}$ has been used. It is well known that the OFDM subcarriers are constant frequency signals. the DFrCT-OCDM subcarrier are chirp like signal as shown in Figure 4 with twice the frequency variations in the DFrFT-OCDM chirp signal which give more frequency diversity and thus potentially more robustness against doubly dispersive channels.

\subsection{The DFrCT-OCDM System}

A conventional OFDM system applies an inverse discrete Fourier transform (IDFT) matrix to a data vector $d_{n}$ and introduces a cyclic prefix (CP) prior to multiplexing to eliminate the ISI. The transmitted OFDM symbol is passed through a dispersive channel $h[m, v]$ corrupted by additive white Gaussian noise $v[n]$. At the receiver, after demultiplexing the received signal and re- 
moving the CP, a DFT matrix reconstructs the transmitted data vector $\hat{d}_{n}$.

In a DFrCT based OCDM system, the DFT matrix is replaced by a DFrCT matrix $F_{\alpha}$ as shown in Figure 5 below:

The subcarriers for the OCDM system are modulated by the IDFrCT where the transmitted data vector $d_{n}=\left[d_{0} d_{1} \cdots d_{N_{a}-1}\right]^{\mathrm{T}}$ and we additionally introduce a binary matrix $P \in \mathbb{Z}^{N \times N}$ which assigns a data vector $d_{n} \in C^{N_{a}}$ to $N$ subcarriers, of which only $N_{a}$ are active according to Equation (7)

$$
P=\left[0_{N_{a} \times\left(N-N_{a}\right) / 2} I_{N_{a}} 0_{N_{a} \times\left(N-N_{a}\right) / 2}\right]
$$

where $0_{L \times M}$ is an $L \times M$ matrix with zero entries, and $I_{N_{a}}$ is an $N_{a} \times N_{a}$ identity matrix. The vector $s=\left[s_{\alpha}(0), s_{\alpha}(1) \cdots s_{\alpha}(N-1)\right]^{\mathrm{T}}$ is calculated from Equation (4):

$$
S_{n}=F_{-\alpha} \cdot P \cdot d_{n}
$$

At the receiver after PC removal in Figure 5, the multiplexed signal $r_{n}=H_{n} s_{n}+v_{n}$ is characterised by a system matrix with elements

$$
\left[H_{n}\right]_{i, j}=\left\{\begin{array}{l}
h[n-L+i, i-j] i \geq j \\
h[n-L+i, L+i-j-1] i<j
\end{array}\right.
$$

In stationary channel conditions, $H_{n}$ is circulant, and can be decoupled by the DFT matrix. Received subcarriers are demodulated using the DFrCT

$$
y_{\alpha}=F_{\alpha} r=F_{\alpha} H s
$$

and the equalizer matrix $W_{n} \in C^{N_{a} \times N_{a}}$ operates on the input:

$$
\hat{r}_{n}=P^{H} F_{\alpha} H_{n} F_{-\alpha} \cdot P \cdot d_{n}+P^{H} F_{\alpha} v
$$

with a system matrix $C_{n, \alpha} \in C^{N_{a} \times N_{a}}$. The binary matrix $P$ is purposed to operate as frequency guard bands, and hence to eliminate the components that could appear in the upper right and lower left corners $C_{n, \alpha} \quad$ [22].

The equivalent $N_{a} \times N_{a}$ channel matrix in the fractional Cosine domain is given by $\tilde{H}_{\alpha}=F_{\alpha} H F_{-\alpha}$ and $\tilde{v}_{\alpha}=F_{\alpha} v_{\alpha}$ is the noise vector in the fractional domain. $\tilde{H}_{\alpha}$ is a nondiagonal subcarrier channel matrix that introduces ICI, which is the case when the dispersive channel comprises a multipath doubly selective channel that destroys the orthogonality between the subcarriers and introduce power leakage between them. This will make the symbol estimation task particularly complicated requiring a complex equalizer.

\subsection{Equalization in the Fractional Cosine Domain}

Linear MMSE and zero-forcing (ZF) estimates [22] may be expressed as follows in Equation (12):

$$
\begin{gathered}
\hat{d}_{\text {MMSE }}=\tilde{H}_{\alpha}^{H}\left(\tilde{H}_{\alpha} \tilde{H}_{\alpha}^{H}+\gamma^{-1} I_{N_{a}}\right)^{-1} y_{\alpha} \\
\hat{d}_{\text {MMSE }}=\tilde{H}_{\alpha}^{+} y_{\alpha}
\end{gathered}
$$

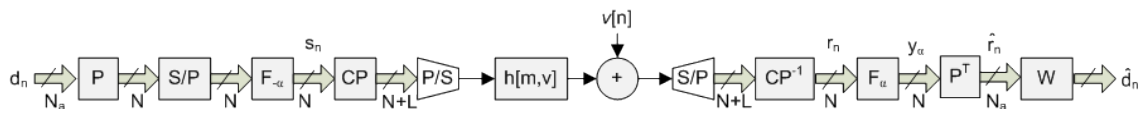

Figure 5. The DFrCT-OCDM system. 
where $\hat{d}_{\text {MMSE }}$ is estimated data after MMSE equalization, $I_{N_{A}}$ is identity matrix with $N_{a} \times N_{a}$ elements, $\gamma$ is the signal-to-noise ratio (SNR) and $\tilde{H}_{\alpha}^{+}$is the Moore-Penrose pseudo-inverse of the channel matrix in the fractional cosine domain [22]. In Equations (12) and (13) perfect knowledge of the channel matrix $\mathrm{H}$ is assumed and the equalizer does not use guard subcarriers. Furthermore it is assumed that $E\left\{d^{(i)}\right\}=E\left\{n^{(i)}\right\}=0$,

$$
E\left\{d^{(i)} d^{(i) H}\right\}=I, \quad E\left\{d^{(i)} n^{(i) H}\right\}=0, \quad E\left\{n^{(i)} n^{(i) H}\right\}=\sigma^{2} I
$$

ZF equalizer performance is poor due to noise enhancement while the MMSE equalizer is particularly complex due to channel matrix inversion which requires $\mathcal{O}\left(N_{v}^{3}\right)$ flops. This is not practical for high values of $N_{A}$. Low complexity equalizers were considered in [23] [24] [25] [26] [27], however, this is beyond this paper work though it is among future work plan.

\subsection{The Selection of Optimal Order $\alpha$}

We will now investigate the effect of the fractional order $\alpha$ on the DFrCTOCDM multicarrier system performance. To improve the multicarrier system performance, $\alpha$ should be chosen to ensure the best match between the subchannel carrier frequencies variation and the fast time-frequency distortion of the channel.

Selecting $\alpha$ depends on the number of subcarriers $\mathrm{N}$, time sample interval Ts, the Doppler shift $f_{D}$, the number of resolvable channel paths and the channel power delay profile. Offline optimization of $\alpha$ for DFrFT-OCDM was proposed in [11] using calculations of the channel statistical expectations. The same method can be used with the DFrCT-OCDM by extracting the channel properties at the receiver and define the optimum $\alpha$ which give the lowest ICI then feedback the $\alpha$ value to the transmitter.

In this paper, we believe that it is extremely important to determine the optimum fractional angle $\alpha$, however, there is no need to repeat the work in [11]. It is important to mention that the used angle fraction is selected to be optimum with the parameters used in this paper based on [11], which means, we believe that there is no need to investigate this point any further.

\section{Performance Analysis}

The uncoded bit error rate (BER) performance of the OFDM, DFrFT-OCDM and the proposed DFrCT-OCDM with the same block MMSE equalizer are investigated by means of simulation. The following system parameters are used: $\mathrm{N}=128, \mathrm{NA}=96, \mathrm{~L}=8, \alpha=0.5 \pi / 2 \quad$ (from offline optimization search) and QPSK modulation is assumed. Rayleigh fading channels with exponential power delay profile, and Jakes' Doppler spectrum. The root-mean-square delay spread of the channel, normalized to the sampling period $T_{S}$, is $\sigma=3$ with maximum Doppler frequency $f_{D}=0.15 \Delta f$. The carrier frequency is $f_{C}=10 \mathrm{GHz}$ and the subcarrier spacing is $\Delta f=20 \mathrm{kHz}$. This Doppler frequency corresponds to a high mobile speed $V=324 \mathrm{Km} / \mathrm{h}$. 
Figure 6 shows the performance of an uncoded BER averaged over 10,000 multicarrier blocks, from which it can be seen that there is an improvement in the performance of the DFrCT-OCDM system compared to both the OFDM and FrFT-OCDM systems with complexity less than the FrFT-OCDM system. From Figure 6, it is observed that the proposed DFrCT at 10-3 Bit Error Rate provides approximately $10 \mathrm{~dB}$ better performance than the OFDM and about $1 \mathrm{~dB}$ improvements over the FrFT-OCDM system and at 10-4 Bit Error Rate provides approximately $3 \mathrm{~dB}$ improvements over the FrFT-OCDM system.

Same results are obtained when using the low comlexity $L D L^{H}$ factorization equalizer.

DFrCT-OCDM System Performance Using the Low complexity $L D L^{\mathrm{H}}$ factorization equaliser:

The low complexity $\mathrm{LDL}^{H}$ factorization equaliser is used to shown the good performance it provides with the DFrFT-OCDM system, The $\mathrm{LDL}^{H}$ factorization equaliser can be used with the DFrCT-OCDM system using the same proposed technique. The uncoded BER performance of the DFrCT-OCDM compared to the OFDM system is investigated by means of simulation over 100000 multicarrier blocks. An FrCT-OCDM system with $\mathrm{N}=128, \mathrm{NA}=96, \mathrm{~L}=8$, and QPSK modulation is assumed. The channel simulation parameters are the same as the Rayleigh fading channel used in the clock MMSE equaliser in Figure 6.

Figure 7 shows that the DFrCT-OCDM system performance is superior to the conventional OFDM system with the same low complexity equaliser. In [28] Cancellation Schemes for BER Performance Improvement of OFDM System is shown, and the itsresults can be compared with these results to show the improvement achieved by DFrCT-OCDM.

DFrCT-OCDM System Performance Using the Low complexity LSMR Equaliser:

The low complexity LSMR equalizer shows almost the same performance as the block MMSE equalizerequaliser in Figure 6 and the LDL $^{H}$ factorization

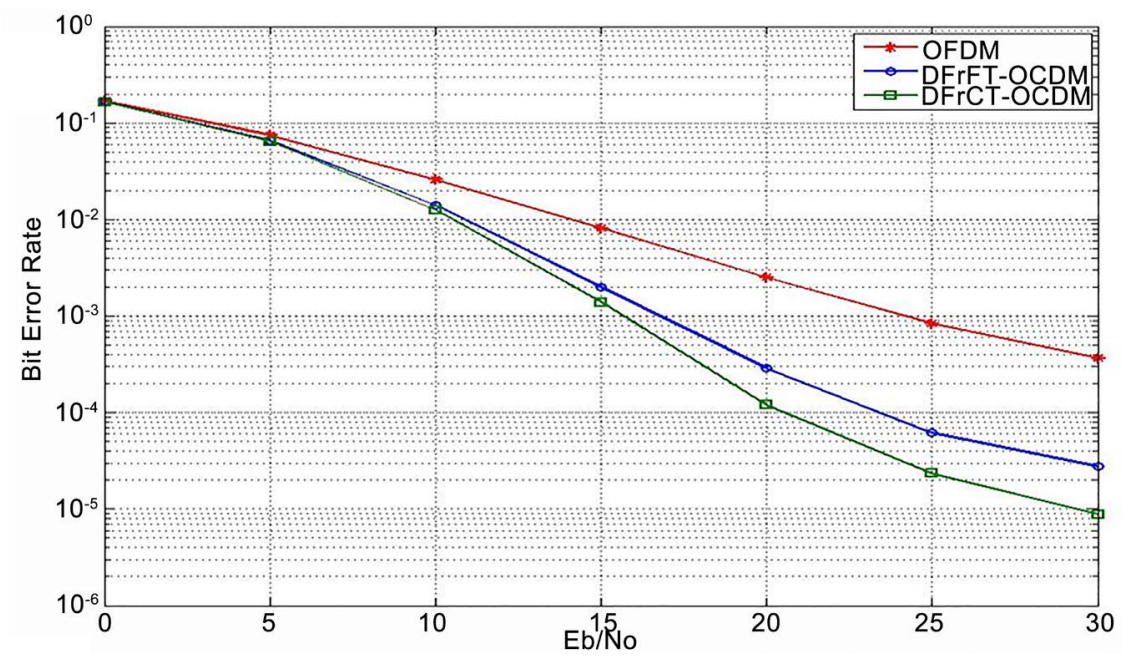

Figure 6. The BER Comparison between DFrCT, DFrFT-OCDM and OFDM using block MMSE equalizer. 




Figure 7. The BER Comparison between DFrCT-OCDM and OFDM using the LDL $^{H}$ factorization equalizer.

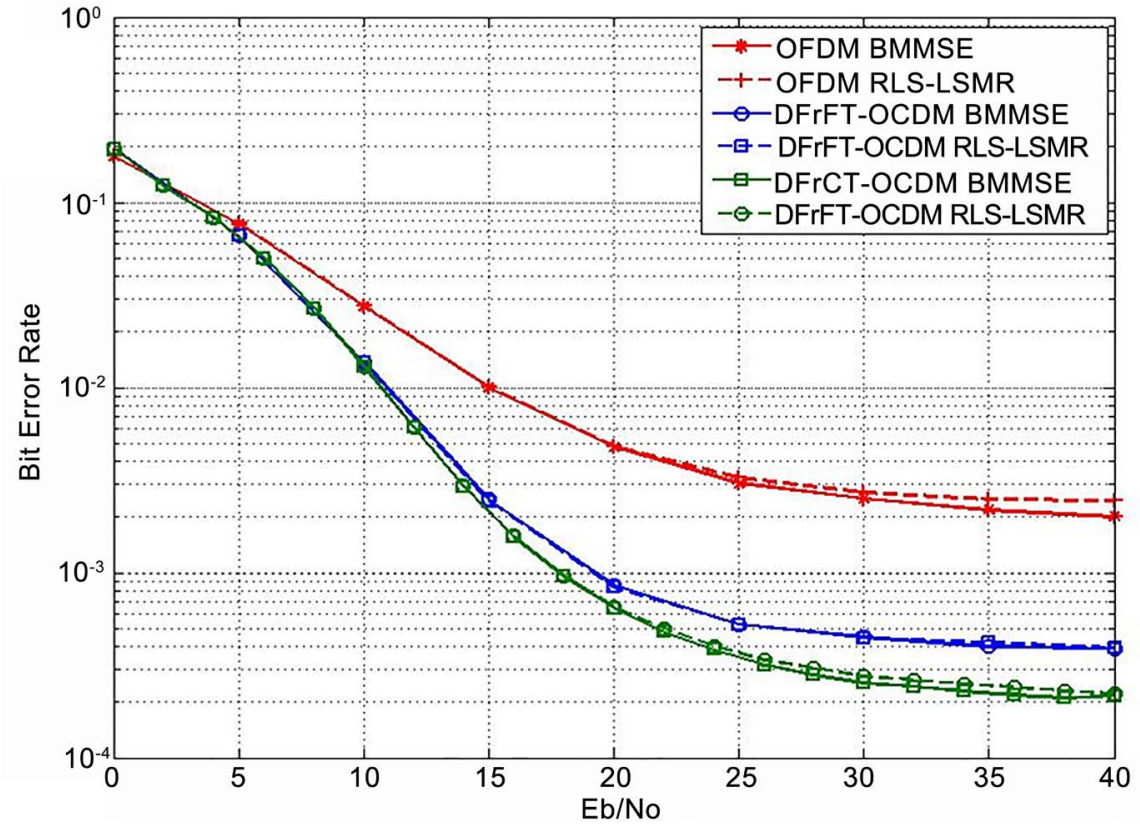

Figure 8. The BER Comparison between DFrCT-OCDM and OFDM using the RLSLSMR equalizer.

equaliserin Figure 7, but with lower complexity. LSMR equaliser can be used with the DFrCT-OCDM following the same technique that was followed with the DFrFT-OCDM.

Figure 8 indicates that the DFrCT-OCDM system performance is better than both the conventional OFDM system and the DFrFT-OCDM system with the same low complexity equaliser.

The different equaliser techniques that are based on the LSMR method can be used all with the DFrCT-OCDM system and it is predicted that the DFrCTOCDM system will be better than the DFrFT-OCDM system and the OFDM 
systems.

\section{Conclusions}

A novel multicarrier transceiver based on the Discrete Fractional Cosine Transform (DFrCT) was proposed. The DFrCT is an optimal modulation/demodulation basis when the multicarrier system faces doubly selective fading channels. It was shown that the DFrCT-OCDM multicarrier system BER performance is superior to the OFDM and the FrFT-OCDM systems with less computational complexity which make the DFrCT a viable multicarrier transceiver in some practical applications that need low power consumption that can be effective in high speed requirement conditions such as DVB-H and WiMAX.

Our ongoing research includes using of low complexity equalizers, testing the peak to average power ratio, time-frequency synchronization and the anti-jamming properties of the new system.

The future work will be directed to investigate the most proper equalizer for the proposed system; moreover, it is important to investigate in more details the effect changing the guard bank with the operation frequency as the basic parameter to mitigate the effect of the Doppler frequency.

Moreover, as total new direction; it is important to go for the downlink communication to investigate this system more with high performance encoders and the ability to apply the new techniques over this system such as Network Coding as it is a band width efficient technique and could help to solve the inefficiency of the bandwidth when increasing the guard band to decrease ICI as a result of the Doppler frequency.

\section{Acknowledgements}

Philadelphia university deserves my true acknowledgements for the good atmosphere they maintain for their researchers and for the financial support for this research.

Moreover, I am always thankful for Dr. Lina Stankovic and Dr. Vladimir Stakovic from the University of Strathclyde, Glasgow, UK, for their support with the technical issues, beside their proof reading and technical modifications in my whole research work. They always add good values to my research work.

\section{References}

[1] Prasad, R. (2004) OFDM for Wireless Communications Systems. Artech House, Norwood.

[2] Molisch, A.F. (2005) Wireless Communications. John Wiley \& Sons, Ltd., Hoboken.

[3] Robertson, P. and Kaiser, S. (1999) Analysis of the Loss of Orthogonality through Doppler Spread in OFDM Systems. Global Telecommunications Conference, Vol. 1b, Rio de Janeireo, 5-9 December 1999, 701-706. https://doi.org/10.1109/glocom.1999.830152

[4] $\mathrm{Li}, \mathrm{W}$. and $\mathrm{Hu}, \mathrm{A}$. (2003) Analyze of the Interchannel Interference of OFDM in Time-Varying Channel. Proceedings of the 2003 International Conference on Neural Networks and Signal Processing, Vol. 1, Nanjing, 14-17 December 2003, 
845-847.

[5] Cai, X. and Giannakis, G.B. (2003) Bounding Performance and Suppressing Intercarrier Interference in Wireless Mobile OFDM. IEEE Transactions on Communications, 51, 2047-2056. https://doi.org/10.1109/TCOMM.2003.820752

[6] Ye, L. and Cimini, L.J.J. (2001) Bounds on the Interchannel Interference of OFDM in Time-Varying Impairments. IEEE Transactions on Communications, 49, 401404. https://doi.org/10.1109/26.911445

[7] Stojanovic, D., et al. (2009) Interference Analysis of Multicarrier Systems Based on Affine Fourier Transform. IEEE Transactions on Wireless Communications, 8, 2877-2880. https://doi.org/10.1109/TWC.2009.081589

[8] Wang, T., Proakis, J.G., Masry, E. and Zeidler, J.R. (2006) Performance Degradation of OFDM Systems Due to Doppler Spreading. IEEE Transactions on Wireless Communications, 5, 1422-1432. https://doi.org/10.1109/TWC.2006.1638663

[9] Robertson, P. and Kaiser, S. (1999) The Effects of Doppler Spreads in OFDM(A) Mobile Radio Systems. 50th Vehicular Technology Conference, Vol. 1, Amsterdam, 19-22 September 1999, 329-333.

[10] Li, J. and Kavehrad, M. (1999) Effects of Time Selective Multipath Fading on OFDM Systems for Broadband Mobile Applications. Communications Letters, 3, 332-334. https://doi.org/10.1109/4234.809526

[11] Martone, M. (2001) A Multicarrier System Based on the Fractional Fourier Transform for Time-Frequency-Selective Channels. IEEE Transactions on Communications, 49, 1011-1020. https://doi.org/10.1109/26.930631

[12] Chen, E., Tao, R. and Meng, X. (2006) The OFDM System Based on the Fractional Fourier Transform. 1st International Conference on Innovative Computing, Information and Control, Beijing, 30 August-1 September 2006, 14-17.

https://doi.org/10.1109/icicic.2006.537

[13] Chen, E., Ma, P., Qi, L. and Tao, R. (2009) Multi-Tap Equalization Algorithm for the OFDM System Based on the Fractional Fourier Transform. 4th International Conference on Communications and Networking in China, Xian, 26-28 August 2009, 1-4.

[14] Alieva, T. and Bastiaans, M.J. (2003) Fractional Cosine and Sine Transforms in Relation to the Fractional Fourier and Hartley Transforms. 7 th International Symposium on Signal Processing and Its Applications, Vol. 1, Paris, 1-4 July 2003, 561 564. https://doi.org/10.1109/isspa.2003.1224765

[15] Peled, A. and Ruiz, A. (1980) Frequency Domain Data Transmission Using Reduced Computational Complexity Algorithms. IEEE International Conference on Acoustics, Speech, and Signal Processing, Denver, 9-11 April 1980, 964-967.

[16] Pei, S.-C. and Ding, J.-J. (2002) Fractional Cosine, Sine, and Hartley Transforms. IEEE Transactions on Signal Processing, 50, 1661-1680.

https://doi.org/10.1109/TSP.2002.1011207

[17] Ouyang, X. and Zhao, J. (2016) Orthogonal Chirp Division Multiplexing.

[18] Attar, H. (2016) Peak-to-Average Power Ratio Performance Analysis for Orthogonal Chirp Division Multiplexing Multicarrier Systems Based on Discrete Fractional Cosine Transform. International Journal of Communications, Network and System Sciences, 9, 545-562.

[19] Schnabel, B. (2016) The Descrete Cosine Transform. SIAM Review, 41, 135-147.

[20] Pei, S.-C. and Ding, J.-J. (2001) Fractional, Canonical, and Simplified Fractional Cosine Transforms. 2001 IEEE International Conference on Acoustics, Speech, and Signal Processing, Vol. 6, Salt Lake City, 7-11 May 2001, 3545-3548. 
[21] Wang, J. and Wang, J. (2005) Feature Extraction Method of Fractional Cosine and Sine Transform for Speaker Recognition. 12th IEEE International Conference on Electronics, Circuits and Systems, Gammarth, 11-14 December 2005, 1-4.

[22] Schniter, P. (2004) Low-Complexity Equalization of OFDM in Doubly Selective Channels. IEEE Transactions on Signal Processing, 52, 1002-1011. https://doi.org/10.1109/TSP.2004.823503

[23] Solyman, A. and Wise, J.J.S.S. (2011) Low-Complexity LSMR Equalisation of FrFT-Based Multicarrier Systems in Doubly Dispersive Channels. 2011 IEEE International Symposium on Signal Processing and Information Technology, Bilbao, 14-17 December 2011, 461-465. https://doi.org/10.1109/isspit.2011.6151606

[24] Han, H. and Wu, L. (2010) Low Complexity LSQR-Based Block Decision Feedback Equalizer for OFDM Systems over Rapidly Time-Varying Channels. 2010 International Conference on Communications and Mobile Computing, Shenzhen, 12-14 April 2010, 438-441.

[25] Liu, G., Ju, T., Wang, Z., Chen, W. and Xu, L. (2010) Simple Equalization of OFDM Signal over Doubly Selective Channels. 2010 International Symposium on Intelligent Signal Processing and Communication Systems, Chengdu, 6-8 December 2010, 1-4. https://doi.org/10.1109/ispacs.2010.5704697

[26] Luca Rugini, P.B. and Leus, G. (2006) Low-Complexity Banded Equalizers for OFDM Systems in Doppler Spread Channels. EURASIP Journal on Applied Signal Processing, 2006, Article ID: 67404.

[27] Rugini, L., Banelli, P. and Leus, G. (2005) Simple Equalization of Time-Varying Channels for OFDM. Communications Letters, 9, 619-621. https://doi.org/10.1109/LCOMM.2005.1461683

[28] Sonika, C. and Deepak, S. (2014) Comparison of ICI Cancellation Schemes for BER Performance Improvement of OFDM System. International Review of Applied Engineering Research, 4, 437-444. http://www.ripublication.com/iraer.htm

Submit or recommend next manuscript to SCIRP and we will provide best service for you:

Accepting pre-submission inquiries through Email, Facebook, LinkedIn, Twitter, etc. A wide selection of journals (inclusive of 9 subjects, more than 200 journals)

Providing 24-hour high-quality service

User-friendly online submission system

Fair and swift peer-review system

Efficient typesetting and proofreading procedure

Display of the result of downloads and visits, as well as the number of cited articles

Maximum dissemination of your research work

Submit your manuscript at: http://papersubmission.scirp.org/

Or contact jcc@scirp.org 